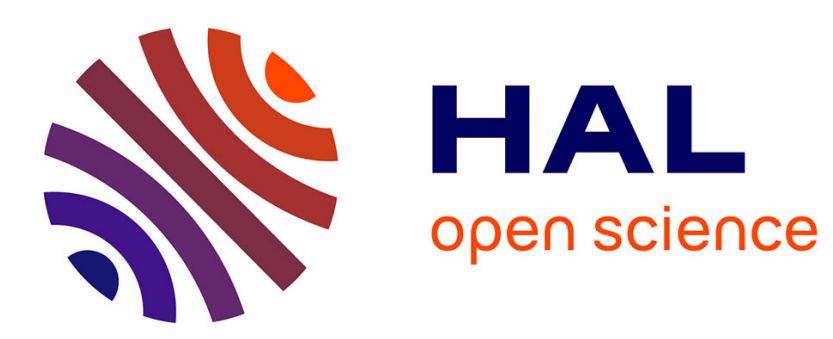

\title{
Polynomial representations of the Hecke algebra of the symmetric group
}

\author{
Alain Lascoux
}

\section{To cite this version:}

Alain Lascoux. Polynomial representations of the Hecke algebra of the symmetric group. International Journal of Algebra and Computation, 2013, 23 (4), pp.803-818. 10.1142/S0218196713400109 . hal00826630

\section{HAL Id: hal-00826630 \\ https://hal.science/hal-00826630}

Submitted on 28 May 2013

HAL is a multi-disciplinary open access archive for the deposit and dissemination of scientific research documents, whether they are published or not. The documents may come from teaching and research institutions in France or abroad, or from public or private research centers.
L'archive ouverte pluridisciplinaire HAL, est destinée au dépôt et à la diffusion de documents scientifiques de niveau recherche, publiés ou non, émanant des établissements d'enseignement et de recherche français ou étrangers, des laboratoires publics ou privés. 


\title{
Polynomial representations of the Hecke algebra of the symmetric group
}

\author{
Alain Lascoux
}

AMS Mathematics Subject Classification: 05E010, 20C08

\begin{abstract}
We give a polynomial basis of each irreducible representation of the Hecke algebra, as well as an adjoint basis. Decompositions in these bases are obtained by mere specializations.
\end{abstract}

\section{Introduction}

Many properties of the algebra of the symmetric group $\mathfrak{S}_{n}$ in characteristic 0 are clarified by having recourse to a $q$-deformation of it, called the Hecke algebra, and denoted $\mathcal{H}_{n}$. Essentially, this algebra is obtained by replacing the quadratic relations $s_{i}^{2}=1$ satisfied by the simple transpositions $s_{i}$, by the relations $\left(s_{i}-q\right)\left(s_{i}+1\right)=0$, or more symmetrically, by $\left(s_{i}-q_{1}\right)\left(s_{i}-q_{2}\right)=0$.

Finding the correct generalizations of Young matrices representing $\mathfrak{S}_{2}$ :

$$
\left[\begin{array}{cc}
g^{-1} & 1-g^{-2} \\
1 & -g^{-1}
\end{array}\right]
$$

was done by Hoefsmit [9], who proposed, for $q_{1}=q, q_{2}=-1$,

$$
\left[\begin{array}{cc}
-\frac{\left(q_{1}+q_{2}\right)}{(\gamma-1)} & -\frac{\left(q_{1} \gamma+q_{2}\right)\left(q_{1}+\gamma q_{2}\right)}{(\gamma-1)^{2}} \\
1 & \frac{\left(q_{1}+q_{2}\right)}{\left(\gamma^{-1}-1\right)}
\end{array}\right],
$$

both parameters $g, \gamma$ being different from 1 .

Young's generators for an irreducible representation of $\mathfrak{S}_{n}$ are made of $2 \times 2$ blocks of the type(1), and similarly matrices made of blocks of the type (2) represent the Hecke algebra. 
A simpler approach to representations of the symmetric group is to find spaces of polynomials in $x_{1}, \ldots, x_{n}$, the simple transpositions $s_{i}$ acting by the exchange of $x_{i}, x_{i+1}$. For example, the linear span of

$$
\left\{\left(x_{1}-x_{2}\right)\left(x_{3}-x_{4}\right),\left(x_{1}-x_{3}\right)\left(x_{2}-x_{4}\right)\right\}
$$

is a 2-dimensional representation of $\mathfrak{S}_{4}$, called Specht representation. However, it is not straightforward that the corresponding space for $\mathcal{H}_{4}$ is the span of

$$
\left(q_{2} x_{1}+q_{1} x_{2}\right)\left(q_{1} x_{4}+q_{2} x_{3}\right) \&\left(q_{2} x_{2}+q_{1} x_{3}\right)\left(q_{1}^{2} x_{4}-q_{2}^{2} x_{1}\right) .
$$

In fact, it is not possible in general to find a basis of polynomials factorizing into linear factors. Moreover, the knowledge of a linear basis is insufficient for most purposes. One usually needs also to be able to decompose any element of the space in this basis. In the case of Specht representations [27], this is insured by the recursive use of straightening relations so much advocated by Gian Carlo Rota [24, 7, 8, 2].

Since straightening relations are a direct consequence of the quadratic Plücker relations between minors of a matrix, one could think of defining a $q$-straightening, and of using it to study representations of the Hecke algebra. However this leads to quantum considerations of much higher complexity than the original work of Young, considerations which cannot be considered as a concrete tool in the theory of representations.

We show in this text that there exist bases of irreducible representations of $\mathcal{H}_{n}$ satisfying easy vanishing properties. In particular, straightening is replaced by mere specializations. We refer to [1] for general considerations on representations, and to [29, 23] and the book [3] for a modern description of the representations of the symmetric group.

\section{$2 \quad$ Hecke algebra and Yang-Baxter relations}

The Hecke algebra $\mathcal{H}_{n}$ is the algebra generated by elements $T_{1}, \ldots, T_{n-1}$ satisfying the braid relations

$$
T_{i} T_{i+1} T_{i}=T_{i+1} T_{i} T_{i+1} \quad \& \quad T_{i} T_{j}=T_{j} T_{i},|i-j| \neq 1,
$$

and the Hecke relations

$$
\left(T_{i}-q_{1}\right)\left(T_{i}-q_{2}\right)=0 .
$$

One can represent faithfully $\mathcal{H}_{n}$ by operators on $\mathfrak{P o l}\left(\mathbf{x}_{n}\right)=\mathbb{C}\left[\left[q_{1}, q_{2}\right]\right]\left(\mathbf{x}_{n}\right)$, writing $\mathbf{x}_{n}=\left\{x_{1}, \ldots, x_{n}\right\}$, such that each $T_{i}$ acts on $x_{i}, x_{i+1}$ only, and commutes with symmetric functions in $x_{i}, x_{i+1}$. Thus $T_{i}$ is represented by an 
operator, still denoted $T_{i}$, which is determined by its action on the basis $\left\{1, x_{i+1}\right\}$ of $\mathfrak{P o l}\left(x_{i}, x_{i+1}\right)$ as a free $\mathfrak{S} \mathfrak{y m}\left(x_{i}, x_{i+1}\right)$-module.

Writing operators on the right, one puts

$$
1 T_{i}=q_{1} \quad \& \quad x_{i+1} T_{i}=-q_{2} x_{i} .
$$

In more details, the operators $T_{i}$ are deformations of Newton's divided differences [15, 16]. Write $f \rightarrow f^{s_{i}}$ for the exchange of $x_{i}, x_{i+1}$ in a polynomial, $\partial_{i}$ for the divided difference $f \rightarrow f \partial_{i}=\left(f-f^{s_{i}}\right)\left(x_{i}-x_{i+1}\right)^{-1}$ and $\pi_{i}=x_{i} \partial_{i}$ for the isobaric divided difference $f \rightarrow f \pi_{i}=\left(x_{i} f-x_{i+1} f^{s_{i}}\right)\left(x_{i}-x_{i+1}\right)^{-1}$. Then

$$
T_{i}=\pi_{i}\left(q_{1}+q_{2}\right)-s_{i} q_{2},
$$

as one can check by using that $1 \pi_{i}=1, x_{i+1} \pi_{i}=0$.

Taking products of simple operators corresponding to reduced decompositions, one obtains operators indexed by permutations [19. In particular, let $\omega=[n, \ldots, 1]$ be the permutation of maximal length in $\mathfrak{S}_{n}$, and $\rho=[n-1, \ldots, 0]$. Then

$$
\partial_{\omega}=\left(\partial_{1} \ldots \partial_{n-1}\right)\left(\partial_{1} \ldots \partial_{n-2}\right) \cdots\left(\partial_{1}\right) \text {. }
$$

This operator has also a global expression given by a summation over the symmetric group [19]:

$$
f \rightarrow f \partial_{\omega}=\left(\sum_{\sigma \in \mathfrak{S}_{n}}(-1)^{\ell(\sigma)} f^{\sigma}\right) \prod_{1 \leq i<j \leq n}\left(x_{i}-x_{j}\right)^{-1} .
$$

From this expression, one sees that when $v \in \mathbb{N}^{n}$ is such that $v_{1}+\cdots+v_{n}=$ $\left(\begin{array}{l}n \\ 2\end{array}\right)$, then one has

$$
x^{v} \partial_{\omega}= \begin{cases}(-1)^{\ell(\sigma)} & \text { if } \exists \sigma: v=\rho \sigma \\ 0 & \text { otherwise }\end{cases}
$$

With products of simple transpositions $s_{i}$, one produces only permutations. It is more fruitful to use the Yang-Baxter relations instead of mere braid relations. For example, if one replaces $s_{1} s_{2} s_{1}$ by $\left(1+s_{1}\right)\left(2^{-1}+s_{2}\right)\left(1+s_{1}\right)$, one obtains by expansion the sum of the 6 permutations of $\mathfrak{S}_{3}$ instead of the permutation of maximal length.

More generally, for $\alpha, \beta$ such that $\alpha, \beta, \alpha \beta \neq 1$, and $i=1, \ldots n-1$, the equalities

$$
\begin{aligned}
\left(T_{i}+\frac{q_{1}+q_{2}}{\alpha-1}\right)\left(T_{i+1}\right. & \left.+\frac{q_{1}+q_{2}}{\alpha \beta-1}\right)\left(T_{i}+\frac{q_{1}+q_{2}}{\beta-1}\right) \\
& =\left(T_{i+1}+\frac{q_{1}+q_{2}}{\beta-1}\right)\left(T_{i}+\frac{q_{1}+q_{2}}{\alpha \beta-1}\right)\left(T_{i+1}+\frac{q_{1}+q_{2}}{\alpha-1}\right)
\end{aligned}
$$


are called Yang-Baxter relations for the Hecke algebra.

These relations can be displayed graphically as follows, writing on the right the corresponding ones for the symmetric group, and taking $i=1$ :
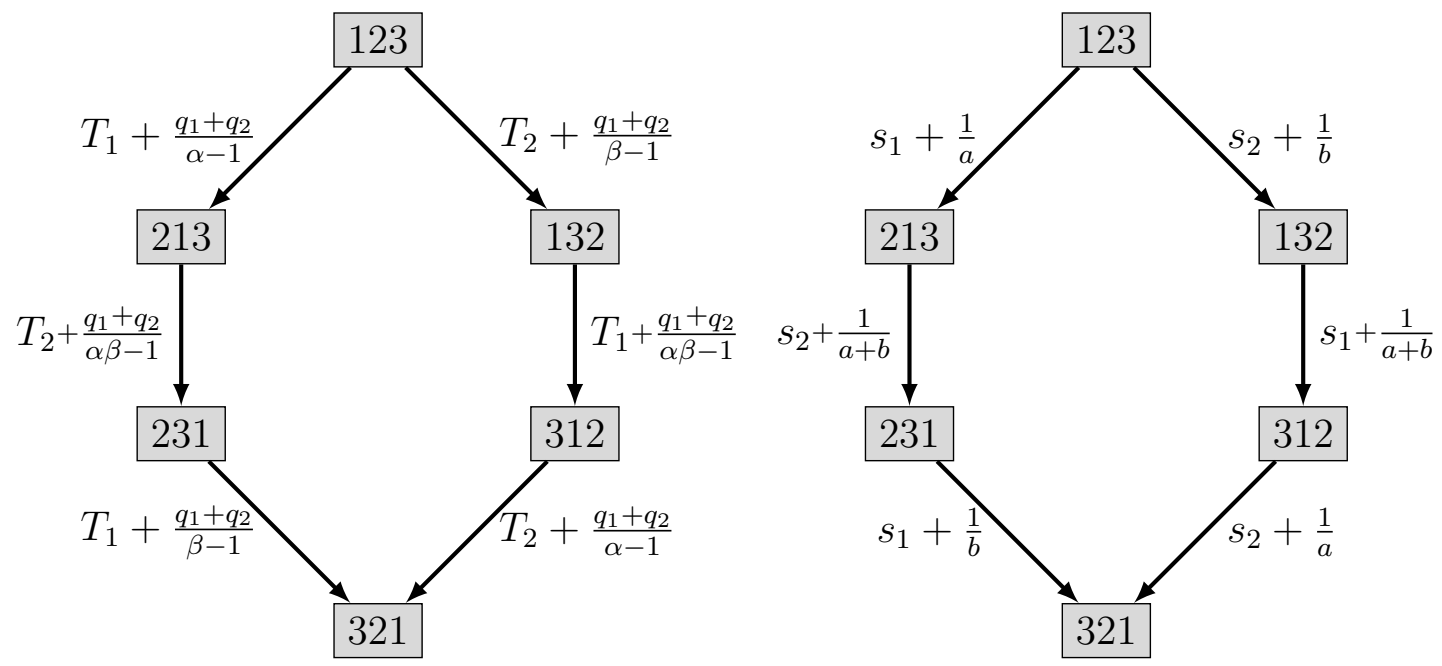

These pictures encode the fact that the two paths from top to bottom evaluate to the same element, when taking the product of the labels in the Hecke algebra or the group algebra respectively.

\section{Yang-Baxter graphs and representations}

Young's semi normal representations of the symmetric group can also be described in terms of a graph. Instead of labeling the vertices by standard Young tableaux in $\mathfrak{T} \mathfrak{a} \mathfrak{b}(\lambda)$, the set of standard tableaux of column-shape $\lambda$, let us directly take content vectors.

Given a partition $\lambda=\left[\lambda_{1}, \ldots, \lambda_{\ell}\right]$ of $n$, let

$$
v^{\lambda}=\left[0,-1, \ldots,\left(-\lambda_{1}+1\right), 1,0, \ldots,\left(-\lambda_{2}+2\right), \ldots,\left(\lambda_{\ell}-1\right), \ldots,\left(-\lambda_{\ell}+\ell\right)\right]
$$

One generates a directed labelled graph $\Gamma_{\lambda}^{\mathfrak{S}}$, starting from $v^{\lambda}$, by the rule: at a vertex $v$, for each $i$ such that $v_{i}<v_{i+1}-1$, there is an ensuing edge of label $s_{i}+\left(v_{i}-v_{i+1}\right)^{-1}$, the new vertex being labelled $v s_{i}$, i.e. being obtained by exchanging the $i$-th and $(i+1)$-th component of $v$. The vectors such obtained are the content vectors for the representation of index $\lambda$ (corresponding to a Ferrers' diagram given by the lengths of its columns). These vectors are in bijection with the tableaux in $\mathfrak{T} \mathfrak{a} \mathfrak{b}(\lambda)$, being obtained by reading the contents of the boxes of the Ferrers' diagram in the order indicated by the tableau. 
Let us denote their set $\mathcal{C}(\lambda)$. Okounkov and Vershik [23] show that content vectors are forced once one wants to build Gelfand-Tsetlin bases of representations of the symmetric group.

For example, for $\lambda=[3,2]$, one has $v^{\lambda}=[0,-1,-2,1,0]$ and the graph $\Gamma_{32}^{\mathfrak{S}}$ has five vertices (we use $\bar{a}$ instead of $-a$; we give a copy of the graph with tableaux as labels)
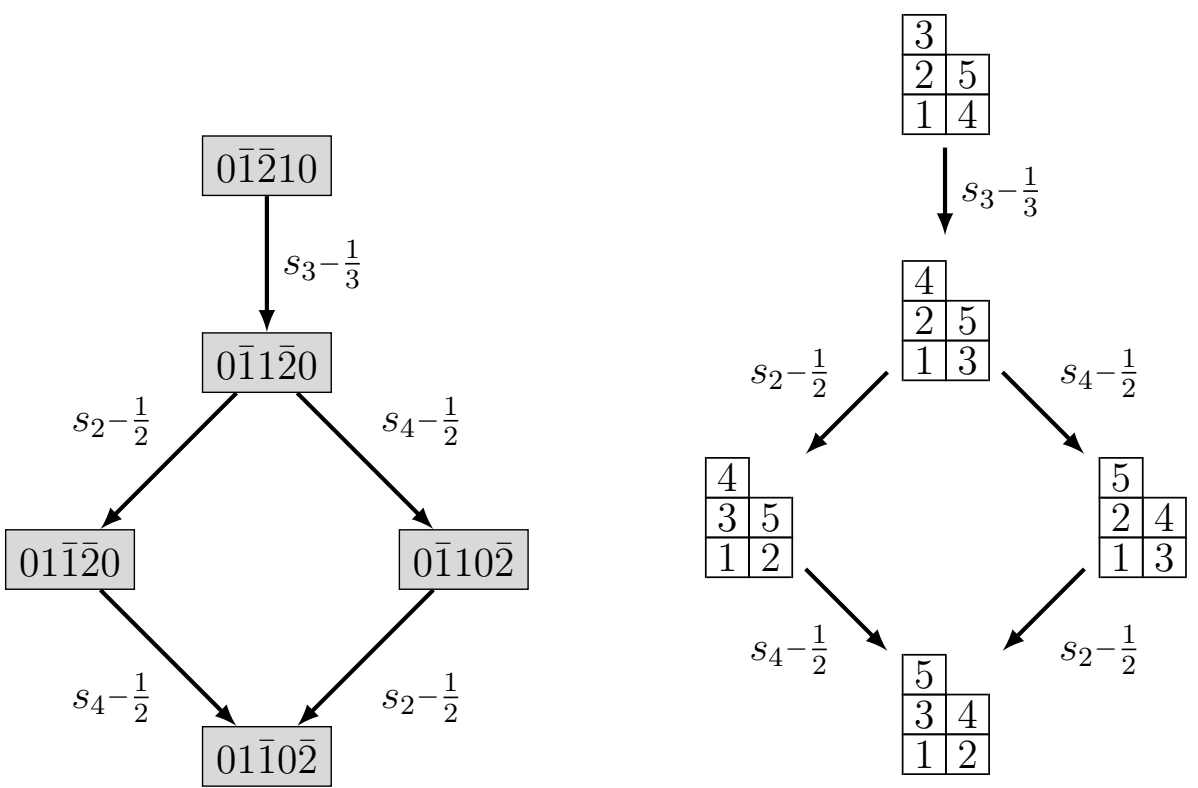

The graph $\Gamma_{\lambda}^{\mathfrak{S}}$ encodes all the matrices representing $s_{1}, \ldots, s_{n-1}$. Indeed, given $i$, one erases all the edges with labels $s_{j}+c$, with $j \neq i$. The graph decomposes into a disjoint union of two-vertices graphs, and singletons. Taking an order on vertices such that vertices connected by an edge are consecutive, one writes a block-diagonal matrix such that each single vertex $v$ with $v_{i}=v_{i+1}+1$ gives a block of size 1 filled with -1 , each vertex with $v_{i}=v_{i+1}-1$ gives a block filled with 1 , a pair $[\ldots, a, b, \ldots] \stackrel{s_{i}+1 /(a-b)}{\longrightarrow}[\ldots, b, a, \ldots]$ is interpreted as above as a matrix $\left[\begin{array}{cc}g^{-1} & 1-g^{-2} \\ 1 & -g^{-1}\end{array}\right]$ with $g=b-a$. These matrices are exactly Young's matrices for semi-normal representations [32, p.451], [25, p.43], [10, p.126]. The fact that the matrices associated to the graph $\Gamma_{\lambda}^{\mathfrak{S}}$ satisfy the braid relations can be reproved directly, and essentially relies on the Yang-Baxter relations. Cherednik explains how to obtain Young idempotents from the Yang-Baxter relations in [4].

It is easy to extend the preceding considerations to representations of the Hecke algebra. Let us now interpret a graph with two vertices $u, v$ and a 
labelled edge

$$
u \stackrel{T_{1}+\frac{q_{1}+q_{2}}{\gamma-1}}{\longrightarrow} v
$$

as a two dimensional representation of $\mathcal{H}_{2}$, with basis $\{u, v\}$ such that $v$ is the image of $u$ under $T_{1}+\frac{q_{1}+q_{2}}{\gamma-1}$. In other words, the matrix representing $T_{1}$ is precisely (2). More generally, the graph $\Gamma_{\lambda}^{\mathcal{H}}$ is defined by changing the labels of $\Gamma_{\lambda}^{\mathfrak{S}}$, keeping the set of vertices $\mathcal{C}(\lambda)$. An edge $v \rightarrow v s_{i}$ is now labelled

$$
T_{i}\left(v_{i}-v_{i+1}\right)=T_{i}+\frac{q_{1}+q_{2}}{\left(-q_{1} / q_{2}\right)^{v_{i}-v_{i+1}}-1} .
$$

For example the graph $\Gamma_{32}^{\mathcal{H}}$ is equal to

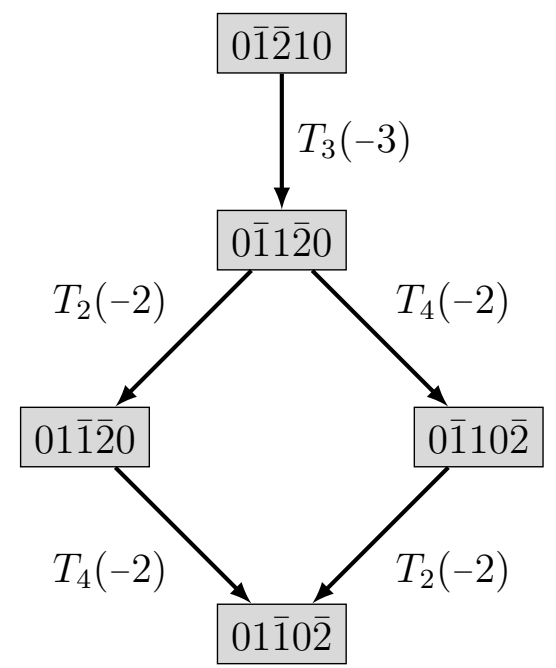

The matrices representing the generators $T_{1}, \ldots, T_{n-1}$ are read from the graph, after adding the rule that a vertex $v$ with $v_{i}=v_{i+1}+1$ (resp. $v_{i}=$ $v_{i+1}-1$ gives a diagonal entry $q_{2}$ (resp. $q_{1}$ ). These matrices are given by Murphy [22], up to a different normalization. Once more, the fact that the graph encodes matrices representing $T_{1}, \ldots, T_{n-1}$ is a consequence of the Yang-Baxter relations [18].

\section{Jucys-Murphy elements}

The sum $\theta_{n}$ of all transpositions in $\mathfrak{S}_{n}$ is a central element in the group algebra (because transpositions constitute a conjugacy class). More generally, identifying $\mathfrak{S}_{k}$ as the subgroup $\mathfrak{S}_{k} \times \mathfrak{S}_{1}$ of $\mathfrak{S}_{k+1}$, one sees that the elements $\theta_{1}, \theta_{2}, \ldots, \theta_{n}$ generate a commutative sub-algebra of $\mathbb{C}\left[\mathfrak{S}_{n}\right]$. The elements 
$0, \theta_{2}, \theta_{3}-\theta_{2}, \ldots, \theta_{n}-\theta_{n-1}$ are called Jucys-Murphy elements. Young's orthogonal idempotents [28, 25] for the symmetric group are eigenfunctions of the Jucys-Murphy elements [11, 22, 3], and this property gives the simplest way to characterize them. Notice nevertheless that multiplying a generic element of the group algebra of the symmetric group by sums of transpositions is a costly operation.

The corresponding Jucys-Murphy elements for $\mathcal{H}_{n}$ can be defined recursively by $\xi_{1}=1$,

$$
\xi_{i}=\frac{-1}{q_{1} q_{2}} T_{i-1} \xi_{i-1} T_{i-1}=1+\left(q_{1}+q_{2}\right) \sum_{j<i}\left(-q_{1} q_{2}\right)^{j-i} T_{(j, i)},
$$

and are still called Jucys-Murphy elements (compared to the preceding case, one adds a constant term to a sum of transpositions $(j, i)$ ). They were in fact first considered by Bernstein. These elements satisfy the following properties which are easy to check [22]. Write $\widehat{T}_{i}=T_{i}-q_{1}-q_{2}$.

Lemma 1 The $\xi_{i}$ generate a commutative algebra and satisfy the relations

$$
\begin{gathered}
\xi_{i} T_{i}=\widehat{T}_{i} \xi_{i+1} \quad \& \quad\left(\xi_{i}+\xi_{i+1}\right) T_{i}=T_{i}\left(\xi_{i}+\xi_{i+1}\right), 1 \leq i \leq n-1 \\
\xi_{j} T_{i}=T_{i} \xi_{j}, j \neq i, i+1
\end{gathered}
$$

The following property allows to generate eigenfunctions of Jucys-Murphy elements, starting from one such element.

Lemma 2 Let $f$ be an eigenvector for the right multiplication by $\xi_{i}$ and $\xi_{i+1}$, with eigenvalues $\alpha, \beta$. Then $g=f\left(T_{i}+\frac{q_{1}+q_{2}}{\alpha / \beta-1}\right)$ is an eigenvector of $\xi_{i}$ and $\xi_{i+1}$ with eigenvalues $\beta, \alpha$.

Proof.

$$
\begin{aligned}
& f\left(T_{i}+\frac{q_{1}+q_{2}}{\alpha / \beta-1}\right) \xi_{i+1}=f\left(\widehat{T}_{i}+\left(q_{1}+q_{2}\right)\left(1+\frac{1}{\alpha / \beta-1}\right)\right) \xi_{i+1} \\
& \quad=f\left(\xi_{i} T_{i}+\xi_{i+1}\left(q_{1}+q_{2}\right) \frac{\alpha / \beta}{\alpha / \beta-1}\right)=f\left(T_{i} \alpha+\left(q_{1}+q_{2}\right) \frac{\alpha}{\alpha / \beta-1}\right)=g \alpha .
\end{aligned}
$$

Since moreover $\xi_{i}+\xi_{i+1}$ commutes with $T_{i}$, the two eigenvalues have been exchanged.

QED

In the algebra generated by the Jucys-Murphy elements, Murphy [22] constructs a set of idempotents $\left\{e_{t}\right\}$ indexed by standard Young tableaux, which are simultaneous eigenfunctions of $\xi_{1}, \ldots, \xi_{n}$. In particular, if $t_{\lambda}$ is the 
tableau of column-shape a partition $\lambda$ filled by consecutive numbers in each column, then the eigenvalues are

$$
\left(-q_{1} / q_{2}\right)^{v_{1}^{\lambda}}, \ldots,\left(-q_{1} / q_{2}\right)^{v_{n}^{\lambda}}
$$

where $v^{\lambda}$ is the content vector defined above. These idempotents generalize Young's orthogonal idempotents.

The space $e_{t_{\lambda}} \mathcal{H}_{n}$ is an irreducible representation of the Hecke algebra, the element $e_{t_{\lambda}}$ being characterized in this space by the set of equations $e_{t_{\lambda}} T_{i}=$ $e_{t_{\lambda}} q_{2}$, for all $i$ such that $s_{i}$ belongs to $\mathfrak{S}_{\lambda}$. For example, for $\lambda=[3,2]$, one has $v^{\lambda}=[0,-1,-2,1,0], t_{32}=$\begin{tabular}{|ll}
\hline 3 & \\
\hline 2 & 5 \\
\hline 1 & 4
\end{tabular}, , the eigenvalues are $1,-q_{2} / q_{1}, q_{2}^{2} / q_{1}^{2},-q_{1} / q_{2}, 1$, and the idempotent $e_{t_{32}}$ satisfies $e_{t_{32}} T_{i}=e_{t_{32}} q_{2}$ for $i=1,2,4$.

Notice that one could have used another set of Jucys-Murphy elements, starting with $\widehat{\xi}_{1}=1$, and defining

$$
\widehat{\xi}_{i}=\frac{-1}{q_{1} q_{2}} \widehat{T}_{i-1} \widehat{\xi}_{i-1} \widehat{T}_{i-1}
$$

This amounts using the involution $T_{i} \rightarrow \widehat{T}_{i}, q_{1} \rightarrow-q_{2}, q_{2} \rightarrow-q_{1}$. The eigenvalues of $\widehat{\xi}_{1}, \ldots, \widehat{\xi}_{n}$ acting on $e_{t_{\lambda}}$ are the inverses of the eigenvalues of $\xi_{1}, \ldots, \xi_{n}$.

Idempotents, one expanded in the basis of permutations, are voluminous expressions. I give a way to encode them formally as factorizing polynomials in [17].

\section{Polynomial representations}

The symmetric group, as well as the Hecke algebra, act on polynomials. Hence representations can be realized as spaces of polynomials.

In the case of the symmetric group, let $\lambda=\left[\lambda_{1}, \ldots, \lambda_{\ell}\right]$ be a partition $n, \mathfrak{S}_{\lambda}=\mathfrak{S}_{\lambda_{1}} \times \ldots \mathfrak{S}_{\lambda_{r}}$ be a Young subgroup. Denote $\mathfrak{P o l}^{d}$ the subspace of $\mathfrak{P o l}\left(x_{1}, \ldots, x_{n}\right)$ of polynomials of degree $d=\left(\begin{array}{c}\lambda_{1} \\ 2\end{array}\right)+\cdots+\left(\begin{array}{c}\lambda_{\ell} \\ 2\end{array}\right)$. The subspace of $\mathfrak{P o l}^{d}$ of polynomials which are alternating under $\mathfrak{S}_{\lambda}$ is 1 -dimensional and generated by the following product of Vandermonde functions

$$
\Delta_{\lambda}=\Delta\left(\left[1, \ldots, \lambda_{1}\right]\right) \Delta\left(\left[\lambda_{1}+1, \ldots, \lambda_{1}+\lambda_{2}\right]\right) \ldots \Delta\left(\left[\lambda_{1}+\ldots+\lambda_{\ell-1}+1, \ldots, n\right]\right)
$$

with $\Delta([a, \ldots, b])=\prod_{a \leq i<j \leq b}\left(x_{j}-x_{i}\right)$. The space $\Delta_{\lambda} \mathbb{C}\left[\mathfrak{S}_{n}\right]$ is an irreducible representation of the symmetric group, called Specht representation [27, 8], [10, Ch. 7]. 
Similarly, the subspace of polynomials $f$ in $\mathfrak{P o l}^{d}$ which are such that $f T_{i}=f q_{2}$ for all $i$ such that $s_{i}$ belongs to $\mathfrak{S}_{\lambda}$, is 1 -dimensional and generated by

$$
\Delta_{\lambda}^{q}=\Delta^{q}\left(\left[1, \ldots, \lambda_{1}\right]\right) \Delta^{q}\left(\left[\lambda_{1}+1, \ldots, \lambda_{1}+\lambda_{2}\right]\right) \ldots \Delta^{q}\left(\left[\lambda_{1}+\ldots+\lambda_{\ell-1}+1, \ldots, n\right]\right)
$$

with $\Delta^{q}([a, \ldots, b])=\prod_{a \leq i<j \leq b}\left(q_{2} x_{i}+q_{1} x_{j}\right)$. Moreover, the space $\Delta_{\lambda}^{q} \mathcal{H}_{n}$ is an irreducible representation of the Hecke algebra.

Though starting points are analogous, the Vandermonde function being replaced by its $q$-generalization, the polynomials obtained under the action of $\mathfrak{S}_{n}$ or $\mathcal{H}_{n}$ look very different. For example, the space $\Delta_{22} \mathbb{C}\left[\mathfrak{S}_{4}\right]$ has basis $\Delta_{22},\left(\Delta_{22}\right)^{s_{2}}=\left(x_{3}-x_{1}\right)\left(x_{4}-x_{2}\right)$, but the polynomial $\Delta_{22}^{q} T_{2}$ is not factorizing, being equal to

$$
-q_{2}^{3} x_{1} x_{2}+q_{1}^{3} x_{3} x_{4}+\left(q_{1}^{2} q_{2}+q_{1}^{3}\right) x_{2} x_{4}+q_{1}^{2} q_{2} x_{1} x_{4}+q_{1}^{2} q_{2} x_{2} x_{3} .
$$

We shall nevertheless see that the Yang-Baxter graph still provides a basis which, though not factorizing, satisfies enough vanishing conditions to characterize it.

Let us first go back to a 2-dimensional representation of $\mathcal{H}_{2}$. Instead of a formal pair $u, v$, let us take a pair of polynomials $f, g \in \mathfrak{P o l}\left(x_{1}, x_{2}\right)$ such that $g=f\left(T_{1}+\left(q_{1}+q_{2}\right)(\gamma-1)^{-1}\right)$. Then the polynomials $g\left(x_{1}, x_{2}\right), g\left(x_{2}, x_{1}\right)$ are given by the following formula, in terms of $A=f\left(x_{1}, x_{2}\right), B=f\left(x_{2}, x_{1}\right)$ :

$$
\begin{aligned}
& g\left(x_{1}, x_{2}\right)=A \frac{\left(q_{1}+q_{2}\right)\left(\gamma-x_{2} / x_{1}\right)}{(\gamma-1)\left(1-x_{2} / x_{1}\right)}+B \frac{q_{2} x_{1}+q_{1} x_{2}}{x_{2}-x_{1}} \\
& g\left(x_{2}, x_{1}\right)=A \frac{q_{1} x_{1}+q_{2} x_{2}}{x_{1}-x_{2}}+B \frac{\left(q_{1}+q_{2}\right)\left(\gamma-x_{1} / x_{2}\right)}{(\gamma-1)\left(1-x_{1} / x_{2}\right)} .
\end{aligned}
$$

Evidently, if $A=0=B$, then $g\left(x_{1}, x_{2}\right)=0=g\left(x_{2}, x_{1}\right)$. For $A \neq 0$ and $B=0$, one has the following property.

Lemma 3 Let $f \in \mathfrak{P o l}\left(x_{1}, x_{2}\right), \alpha, \beta \in \mathbb{C}$ be such that $\alpha \neq \beta, q_{1} \alpha+q_{2} \beta \neq 0$ and $f(\alpha, \beta) \neq 0, f(\beta, \alpha)=0$. Let moreover $g=f\left(T_{1}+\left(q_{1}+q_{2}\right)(\beta / \alpha-1)^{-1}\right)$. Then

$$
g(\alpha, \beta)=0 \quad \& \quad g(\beta, \alpha)=\frac{q_{1} \alpha+q_{2} \beta}{\alpha-\beta} f(\alpha, \beta) .
$$

In the case of the representation of $\mathcal{H}_{2}$ generated by the action of $T_{2}$ on $\Delta_{22}^{q}$, one sees that $\Delta_{22}^{q}$ vanishes in the point $p_{2}=\left[1,-q_{2} / q_{1},-q_{1} / q_{2}, 1\right]$, but not in $p_{1}=\left[1,-q_{1} / q_{2},-q_{2} / q_{1}, 1\right]$. The lemma implies that $f=\Delta_{22}^{q}\left(T_{2}+\right.$ 
$\left.\left(q_{1}+q_{2}\right)\left(\left(-q_{2} / q_{1}\right)^{2}-1\right)^{-1}\right)$ vanishes in the point $p_{1}$ and not in $p_{2}$. The explicit value of $f$ is

$$
f=\frac{q_{1}^{2} q_{2}^{2}}{q_{1}-q_{2}}\left(\frac{q_{2}\left(q_{1}-q_{2}\right) x_{1} x_{2}}{q_{1}^{2}}-\frac{q_{1}\left(q_{1}-q_{2}\right) x_{3} x_{4}}{q_{2}^{2}}+\left(x_{1}+x_{2}\right)\left(x_{3}+x_{4}\right)\right)
$$

an expression which looks rather complicated for just studying a 2-dimensional space. We shall see in the next section that the graph $\Gamma_{\lambda}$ provides polynomials having the required vanishing conditions. In fact, Sahi [26] and Knop [14] use a similar construction to define non-symmetric non-homogeneous Macdonald polynomials, we have rephrased it in terms of Yang-Baxter graphs in [18.

\section{Young's basis}

Let us define $q$-integers $[r]$ by $[0]=0$ and, for $r \in \mathbb{N} \backslash 0$,

$$
[r]=q_{1}^{r-1}-q_{1}^{r-2} q_{2}+\cdots+\left(-q_{2}\right)^{r-1} .
$$

To $v \in \mathbb{N}^{n}$, one associates the vector $\langle v\rangle=\left[\left(-q_{2} / q_{1}\right)^{v_{1}}, \ldots,\left(-q_{2} / q_{1}\right)^{v_{n}}\right]$.

To define Young's basis for the Hecke algebra, we keep the vertices $\mathcal{C}(\lambda)$ of the graph $\Gamma_{\lambda}^{\mathcal{H}}$, but change the labeling of edges by normalization factors. With $r=v_{i+1}-v_{i}$, an edge $v \rightarrow v s_{i}$ is labelled

$$
\frac{[r-1]}{[r]} T_{i}(-r)=\frac{[r-1]}{[r]}\left(T_{i}+\frac{q_{1}+q_{2}}{\left(-q_{1} / q_{2}\right)^{r}-1}\right) .
$$

Finally, we interpret the vertices as indexing polynomials, starting from $\Delta_{\lambda}^{q}$, using the edges as operators, to generate polynomials $Y_{v}$ from top to bottom. The Yang-Baxter relations are still preserved, so that two paths with the same end points define the same polynomial. We call this set of polynomials Young's basis, because these polynomials (we shall see that they are linearly independent) are either preserved or annihilated by the the idempotents generalizing Young's orthogonal idempotents. To the set of vertices $\{v\}$ we associate a second set $S p(\lambda)=\{\langle v\rangle\}$ that we call the set of spectral vectors (they are the eigenvalues with respect to $\widehat{\xi}_{1}, \ldots, \widehat{\xi}_{n}$ ). 
For example, for $\lambda=[3,2]$, the graph $\Gamma_{32}^{\mathcal{H}}$ is

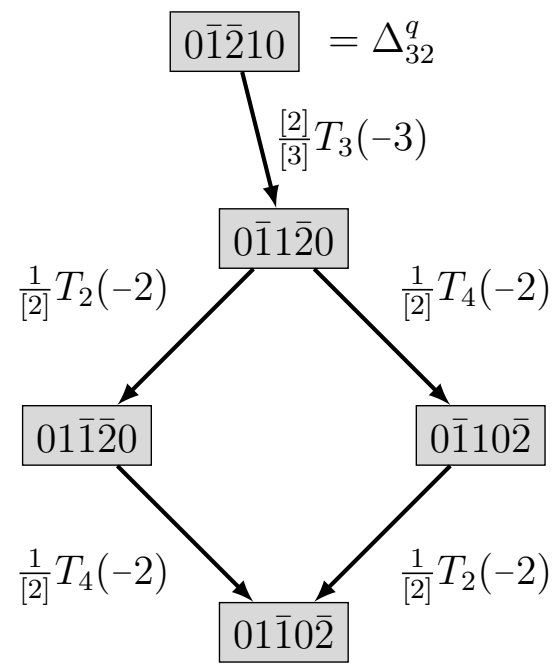

and the spectral vectors are $\left[1,-\frac{q_{1}}{q_{2}}, \frac{q_{1}^{2}}{q_{2}^{2}},-\frac{q_{2}}{q_{1}}, 1\right],\left[1,-\frac{q_{1}}{q_{2}},-\frac{q_{2}}{q_{1}}, \frac{q_{1}^{2}}{q_{2}{ }^{2}}, 1\right],\left[1,-\frac{q_{1}}{q_{2}},-\frac{q_{2}}{q_{1}}, 1, \frac{q_{1}{ }^{2}}{q_{2}{ }^{2}}\right]$, $\left[1,-\frac{q_{2}}{q_{1}},-\frac{q_{1}}{q_{2}}, \frac{q_{1}^{2}}{q_{2}^{2}}, 1\right],\left[1,-\frac{q_{2}}{q_{1}},-\frac{q_{1}}{q_{2}}, 1, \frac{q_{1}^{2}}{q_{2}^{2}}\right]$.

We are finally in position to conclude.

Theorem 4 Let $\lambda$ be a partition, and $\left\{Y_{v}: v \in \mathcal{C}(\lambda)\right\}$ be the set of polynomials obtained from the graph $\Gamma_{\lambda}^{\mathcal{H}}$ as explained above. Let

$$
c_{\lambda}=q_{1}^{\left(\begin{array}{c}
n \\
3
\end{array}\right)} q_{2}^{-n(n-1)(2 n-1) / 6}[2]^{n-1}[3]^{n-2} \ldots[n] .
$$

Then, the polynomials $\left\{Y_{v}\right\}$ constitute a basis of an irreducible representation of $\mathcal{H}_{n}$ and, for any content vector $u \in \mathcal{C}(\lambda)$, one has

$$
Y_{v}(\langle u\rangle)=\delta_{v, u} c_{\lambda}
$$

Each $Y_{v}$ is a simultaneous eigenfunction of the Jucys-Murphy elements,

$$
Y_{v} \xi_{i}=Y_{v}\left(-q_{2} / q_{1}\right)^{v_{i}^{\lambda}} \quad \& \quad Y_{v} \widehat{\xi}_{i}=Y_{v}\left(-q_{1} / q_{2}\right)^{v_{i}^{\lambda}}
$$

Proof. The $q$-Vandermonde $\prod_{i<j}\left(q_{2} x_{i}+q_{1} x_{j}\right)$ vanishes if some $x_{j}$ is specialized to $\left(-q_{2} / q_{1}\right) x_{i}$. Accordingly, $\Delta_{\lambda}$ vanish on a spectral vector $\langle v\rangle$ if, cutting $v$ into blocks of successive lengths $\lambda_{1}, \lambda_{2}, \ldots$ there are two components of $v$ inside a block such that $v_{j}-v_{i}=1, j>i$. But this is the case of all content vectors, except the first one. The evaluation of $\Delta_{\lambda}$ in the first spectral vector is equal to $c_{\lambda}$. The lemma 3 propagates the fact that each polynomial $Y_{v}$ vanishes in every spectral vector, except $\langle v\rangle$. The normalization factors have 
been chosen in such a way that the non-zero specializations are all equal. This proves (12).

The space $\mathfrak{P o l}^{d} e_{t_{\lambda}} \mathcal{H}_{n}$ coincides with the space $\Delta_{\lambda}^{q} \mathcal{H}_{n}$. Since both $e_{t_{\lambda}}$ and $\Delta_{\lambda}^{q}$ are annihilated by the $T_{i}-q_{2}$ for all $i$ such that $s_{i}$ belongs to $\mathfrak{S}_{\lambda}$, the polynomial $\Delta_{\lambda}^{q}$ must belong to the space $\mathfrak{P o l}^{d} e_{t_{\lambda}}$. This implies that $\Delta_{\lambda}^{q}$ is an eigenfunction of the Jucys-Murphy elements, the eigenvalues of $\widehat{\xi}_{1}, \ldots, \widehat{\xi}_{n}$ being the components of the spectral vector $\left\langle v^{\lambda}\right\rangle$. Lemma 2 implies that the other polynomials $Y_{v}$ are still eigenvalues of the Jucys-Murphy elements. QED

We have used normalization factors $[k-1][k]^{-1}$ to obtain a constant scalar product in (12). In fact, Young defines a "tableau function" $f(t)$ in [32, p.458] , such that the normalization factor specializes, for $q_{1}=1, q_{2}=-1$, to the quotient $f(t) / f\left(t^{\prime}\right)$ for a pair of tableaux differing by a simple transposition (see also [10, p.312], and [25, p.47] for a similar function).

There are other families of polynomials which are generated using the Hecke algebra, in particular the non-symmetric Macdonald polynomials. Let us refer to [6] for the fact that some Macdonald polynomials specialize, up to normalization, to the Young basis for $\lambda=[n, n]$ (the extra parameter $q$ occurring in Macdonald's theory is in that case specialized to $\left.-\left(q_{2} / q_{1}\right)^{3}\right)$.

One can also use the Kazhdan-Lusztig basis of the Hecke algebra to generate polynomials. For example, among the images of $\Delta_{32}^{q}$ under KazhdanLusztig elements, thus in the span of the Young basis for $\lambda=[3,2]$, one finds the two factorizing polynomials

$$
\begin{gathered}
q_{1}\left(q_{1} x_{4}+q_{2} x_{3}\right)\left(q_{1} x_{4}+q_{2} x_{2}\right)\left(q_{2} x_{2}+q_{1} x_{3}\right)\left(q_{1}^{2} x_{5}-q_{2}^{2} x_{1}\right), \\
q_{1}^{2}\left(q_{2} x_{4}+q_{1} x_{5}\right)\left(q_{1} x_{5}+q_{2} x_{3}\right)\left(q_{1} x_{4}+q_{2} x_{3}\right)\left(q_{2} x_{1}+q_{1} x_{2}\right),
\end{gathered}
$$

which are known to physicists [12, 5]. In terms of the Young basis, by computing five specializations, one obtains that the first one is equal to

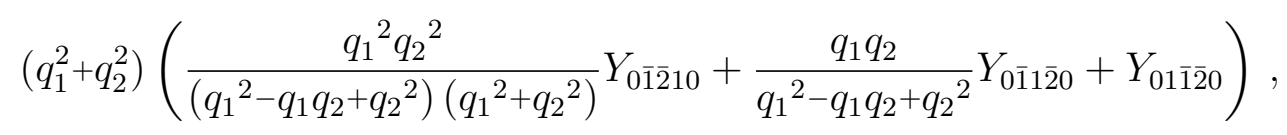

the second one having a similar expression. Computing the action of JucysMurphy elements on these polynomials would be of no use to obtain their decomposition in the Young basis.

Young bases for $\lambda=[n, n]$ and $\lambda=[2, \ldots, 2]$ can be used to compute Pfaffians [20]. 


\section{Adjoint basis}

One can easily build copies of the irreducible representation $\Delta_{\lambda}^{q} \mathcal{H}_{n}$ in higher degree by starting with a polynomial $f \Delta_{\lambda}^{q}$, with $f$ invariant under the Young subgroup $\mathfrak{S}_{\lambda}$. Taking the same Yang-Baxter graph $\Gamma_{\lambda}$, one generates, as in the case $f=1$, a set of polynomials $\left\{Y_{v}^{f}: v \in \mathcal{C}(\lambda)\right\}$ which satisfies the property

$$
Y_{v}^{f}(\langle u\rangle)=\delta_{v, u} c_{\lambda}^{f}, u \in \mathcal{C}(\lambda),
$$

with $c_{\lambda}^{f}=f\left(\left\langle v^{\lambda}\right\rangle\right) c_{\lambda}$. Therefore, any function $f$ invariant under $\mathfrak{S}_{\lambda}$ which does not vanish in $\left\langle v^{\lambda}\right\rangle$ provides a Young basis satisfying the same properties as in Theorem 4 .

Notice also that reversing the directed graph $\Gamma_{\lambda}$, keeping the same labels on edges and starting with the polynomial $\Delta_{\mu}^{q}$, with $\mu=\lambda^{\sim}$, the conjugate partition, labeling the vertices by the elements of $\mathcal{C}(\mu)$, one obtains a basis of the representation $\Delta_{\mu}^{q} \mathcal{H}_{n}$.
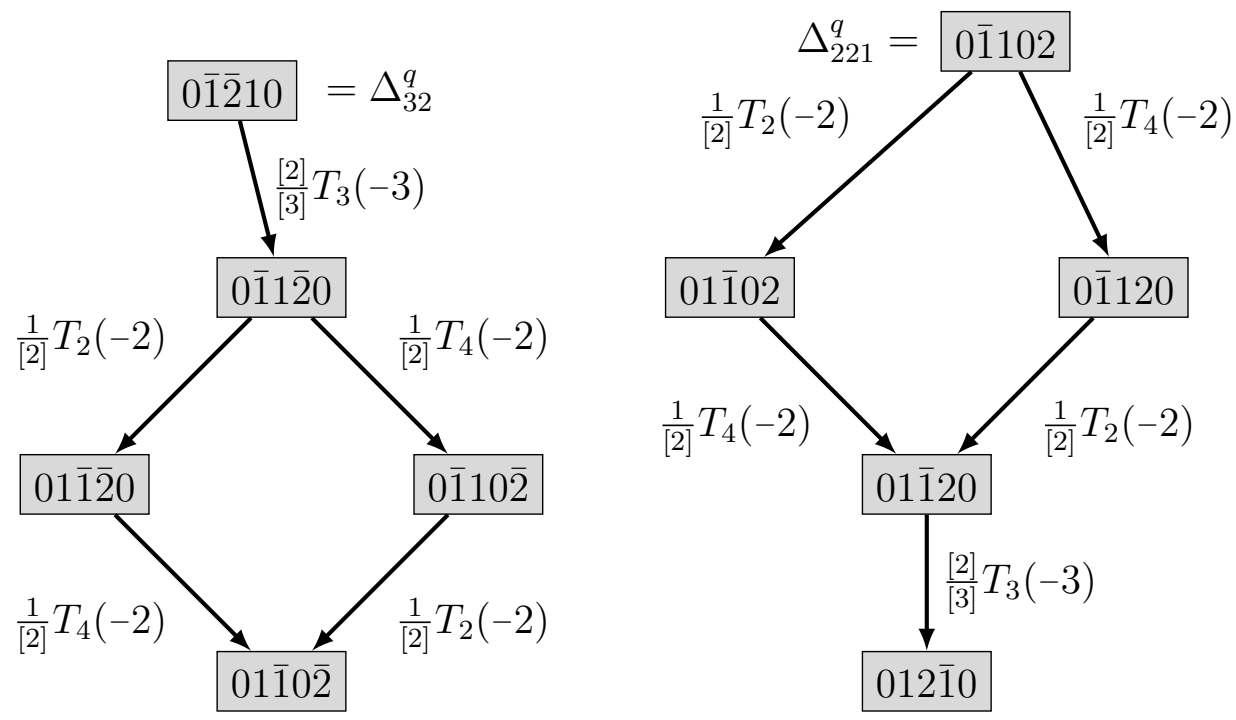

The labels on the vertices are exchanged by $v \rightarrow-v$. In particular, the bottom element of the left graph has label $-v^{\mu}=-[0,-1,1,0,2]=[0,1,-1,0,-2]$.

One can build other bases by having recourse to the work of Kazhdan and Lusztig [13. Kazhdan and Lusztig defined a linear basis $\left\{C_{w}: w \in \mathfrak{S}_{n}\right\}$ of $\mathcal{H}_{n}$. This basis is such that, when $w=\omega_{\lambda}$ is the permutation of maximal length of $\mathfrak{S}_{\lambda}$, then $C_{\omega_{\lambda}}$ can be factorized, as an operator on polynomials, as the product of the divided difference $\partial_{\omega_{\lambda}}$ by $\Delta_{\lambda}^{q}$. In particular, the elements corresponding to simple transpositions $s_{i}$ are such that

$$
C_{s_{i}}=\partial_{i}\left(q_{2} x_{i}+q_{1} x_{i+1}\right)=T_{i}(-1) .
$$


Putting $\rho_{\lambda}=\left[\lambda_{1}-1, \ldots, 0, \lambda_{2}-1, \ldots, 0, \ldots, \lambda_{\ell}-1, \ldots, 0\right]$, one has $x^{\rho_{\lambda}} \partial_{\omega_{\lambda}}=$ 1 , and therefore the module $x^{\rho_{\lambda}} C_{\omega_{\lambda}} \mathcal{H}_{n}$ coincides with the representation that we have studied in the preceding section.

For any standard tableau of (column) shape $\lambda$, denote $\sigma(t)$ the inverse of the permutation obtained by reading the tableau column-wise, from left to right. It results from the work of Kazhdan and Lusztig that the set

$$
\left\{L_{t}=x^{\rho_{\lambda}} C_{\sigma(t)}: t \in \mathfrak{T} \mathfrak{a} \mathfrak{b}(\lambda)\right\}
$$

constitutes a linear basis of the module $x^{\rho_{\lambda}} C_{\omega_{\lambda}} \mathcal{H}_{n}$ (instead of a polynomial representation, Kazhdan and Lusztig [13] take a quotient of $C_{\omega_{\lambda}} \mathcal{H}_{n}$ ). By construction, if $w \in \mathfrak{S}_{n}$ is such that $w_{i}>w_{i+1}$, then $C_{w} C_{s_{i}}=q_{2} C_{w}$. Accordingly, if $i+1$ occurs left of $i$ in the tableau $t$, then

$$
q_{2} L_{t}=L_{t} \partial_{i}\left(q_{2} x_{i}+q_{1} x_{i+1}\right) .
$$

Fill each box of the diagram with row lengths $\lambda$ with the number of boxes in the rows below it. Let $\wp_{\lambda}$ be the column reading of this filling, reading the columns from left to right.

$$
\lambda=[3,2,2], \quad \text { filling } \begin{array}{|l|l|l|}
\hline 5 & 5 & \\
\hline 3 & 3 & \\
\hline 0 & 0 & 0 \\
\hline
\end{array} \quad, \quad \wp_{322}=[5,3,0,5,3,0,0] .
$$

Then $x^{\wp_{\lambda}} \partial_{\omega_{\mu}}$ is a product of Schur functions in blocks of consecutive variables, and therefore the polynomials $\widetilde{Y}_{v}$ generated by the Yang-Baxter graph with top element $\widetilde{\Omega}_{\lambda}=x^{\wp_{\lambda}} C_{\omega_{\mu}}$, for $v$ running over $\mathcal{C}(\mu)$, constitute a Young basis.

Let us show that this basis is adjoint to the previous basis $\left\{Y_{v}: v \in \mathcal{C}(\lambda)\right\}$, with respect to a quadratic form, with values in $\mathfrak{S} \mathfrak{y m}\left(\mathbf{x}_{n}\right)$ (this form occurs in the study of the cohomology ring of the flag variety, [19]). Denote $f \rightarrow f^{q}$ the image of a polynomial under the exchange of $q_{1}, q_{2}$, and

$$
(f, g)=f g^{q} \partial_{\omega} \in \mathfrak{S y m}\left(\mathbf{x}_{n}\right) .
$$

In particular, if the product $f g^{q}$ is homogeneous of degree $\left(\begin{array}{l}n \\ 2\end{array}\right)$, then according to (5), $(f, g)$ is of degree 0 in $\mathbf{x}_{n}$ and its value is obtained by extracting in $f g^{q}$ the monomials which are permuted from $x^{\rho}$.

The following lemma describes the compatibility of the action of the Hecke algebra with the quadratic form.

Lemma 5 Let $f, g \in \mathfrak{P o r}\left(\mathbf{x}_{n}\right), i \in\{1, \ldots, n-1\}, k \geq 1$. Then

$$
\begin{aligned}
\left(f T_{i}(-k), g\right) & =\left(f, g T_{i}(k)\right), \\
\left(f T_{i}(-k) \frac{[k-1]}{[k]}, g\right) & =-\left(f, g T_{i}(k) \frac{[k-1]}{[k]}\right) .
\end{aligned}
$$


Proof. Suppose $k=1$. Then $T_{i}(-1)=\partial_{i}\left(q_{2} x_{i}+q_{1} x_{i+1}\right)$,

$$
\begin{aligned}
& \left(f T_{i}(-1), g\right)=f \partial_{i}\left(q_{2} x_{i}+q_{1} x_{i+1}\right) g^{q} \partial_{\omega}=f \partial_{i}\left(g\left(q_{1} x_{i}+q_{2} x_{i+1}\right)\right)^{q} \partial_{\omega} \\
& \begin{aligned}
\left(g\left(q_{1} x_{i}+q_{2} x_{i+1}\right) \partial_{i}\right)^{q} f \partial_{\omega} & =\left(g \partial_{i}\left(q_{1} x_{i+1}+q_{2} x_{i}\right)+q_{1}-q_{2}\right)^{q} f \partial_{\omega} \\
& =\left(f, g\left(T_{i}-q_{1}+q_{1}-q_{2}\right)\right)=\left(f, g\left(T_{i}(1)\right)\right),
\end{aligned}
\end{aligned}
$$

using the Leibniz's formula $g h \partial_{i}=g \partial_{i} h^{s_{i}}+h \partial_{i} g$. Since

$$
\begin{aligned}
T_{i}(-k) & =T_{i}(-1)-q_{1} q_{2} \frac{[k-1]}{[k]} \\
T_{i}(k) & =T_{i}(1)+q_{1} q_{2} \frac{[k-1]}{[k]}=T_{i}(1)-q_{1} q_{2}\left(\frac{[k-1]}{[k]}\right)^{q},
\end{aligned}
$$

the case $k=1$ implies the case for any $k$, that is $(5)$. The factor $[k-1][k]^{-1}$ creates a sign in the second equation.

Lemma 6 Let $\lambda$ be a partition of $n, \mu=\lambda^{\sim}$. Then $\left(Y_{v}, \widetilde{\Omega}_{\lambda}\right)=0$, except $\left(Y_{-v^{\mu}}, \widetilde{\Omega}_{\lambda}\right)=d_{\lambda}$ for some non-zero function $d_{\lambda}$ of $q_{1}, q_{2}$.

Proof. The Kazhdan-Lusztig basis is triangular in the Young basis, with respect to any order compatible with the graph $\Gamma_{\lambda}^{\mathcal{H}}$. If a tableau $t$ and $i$ are such that $t$ contains the subword $i+1, i$, then according to (15), one has $L_{t}=f\left(q_{2} x_{i}+q_{1} x_{i+1}\right)$, with $f \in \mathfrak{S} \mathfrak{y m}\left(x_{i}, x_{i+1}\right)$. Similarly, if $s_{i} \in \mathfrak{S}_{\mu}$, then $\Delta_{\mu}^{q}=g\left(q_{2} x_{i}+q_{1} x_{i+1}\right)$, with $g \in \mathfrak{S} \mathfrak{y m}\left(x_{i}, x_{i+1}\right)$. In that case, $\left(L_{t}, \Delta_{\mu}^{q}\right)=$ $f g^{q}\left(q_{2} x_{i}+q_{1} x_{i+1}\right)\left(q_{1} x_{i}+q_{2} x_{i+1}\right) \partial_{\omega}$ is null because of the symmetry in $x_{i}, x_{i+1}$. Thus one can expect $\left(L_{t}, \Delta_{\mu}^{q}\right) \neq 0$ only if $t$ contains the subwords $1 \ldots \mu_{1}$, $\left(\mu_{1}+1\right) \ldots\left(\mu_{1}+\mu_{2}\right), \ldots$. In other words $t$ must be the tableau of shape $\lambda$ with rows filled with consecutive integers, denoted $\aleph$. To show that $d_{\lambda}$ is different from 0 , one can specialize $q_{1} \rightarrow 1, q_{2} \rightarrow 0$. The operators $T_{i}(-k)$ tend to $\partial_{i} x_{i+1}=\pi_{i}-1$, and one sees easily that $L_{\aleph}$ specializes to $x^{0^{\mu_{1}} 1^{\mu_{2}} \ldots}$ and that $\widetilde{\Omega}_{\lambda}^{q}$ specializes to $x^{\wp_{\lambda}} \partial_{\omega_{\mu}} x^{\rho_{\mu}}$. A little familiarity with divided differences allows us to conclude that $\left(L_{\aleph}, \widetilde{\Omega}_{\lambda}\right)$ specializes to \pm 1 , which finishes the proof of the lemma.

QED

Combining Lemmas 5 and 6 , denoting now the starting element $\widetilde{\Omega}_{\lambda}$ by $\widetilde{Y}_{v^{\mu}}$, one otains

Theorem 7 Let $\lambda$ be a partition of $n, \mu=\lambda^{\sim}$. Let $\left\{Y_{v}, v \in \mathcal{C}(\lambda)\right\}$ be the Young basis of the module $\Delta_{\lambda}^{q} \mathcal{H}_{n},\left\{\widetilde{Y}_{u}, u \in \mathcal{C}(\mu)\right\}$ be the Young basis of the module $x^{\wp_{\lambda}} C_{\omega_{\mu}} \mathcal{H}_{n}$. Let $\epsilon_{u}= \pm 1$ according to the parity of the distance of $u \in \mathcal{C}(\mu)$ to $v^{\mu}$. Then there exists a non-zero function $d_{\lambda}$ of $q_{1}, q_{2}$ such that

$$
\left(Y_{v}, \widetilde{Y}_{u}\right)=\epsilon_{u} \delta_{v,-u} d_{\lambda}
$$




\section{References}

[1] H. Barcelo, A. Ram. Combinatorial representation theory, New perspectives in algebraic combinatorics, Math. Sci. Res. Inst. Publ., 38, Cambridge Univ. Press, Cambridge (1999) 23-90.

[2] C. Carré, A. Lascoux, B. Leclerc. Turbo straightening, Int J. of Algebra and Comp. (1992) 275-290.

[3] T. Ceccherini-Silberstein, F. Scarabotti, F. Tolli. Representation theory of the symmetric groups, Cambridge Studies in Adv. Math. 121, Cambridge University Press (2010).

[4] I. Cherednik. On special bases of irreducible finite-dimensional representations of the degenerate affine Hecke algebra, Funct. Anal. Appl. 20 (1986) 87-89.

[5] P. Di Francesco, P. Zinn-Justin. Around the Razumov-Stroganov conjecture: proof of a multi-parameter sum rule, Electr. J. Comb., 12: R6 (2005).

[6] Jan de Gier, Alain Lascoux, Mark Sorrell. Deformed Kazhdan-Lusztig elements and Macdonald polynomials, J. of Comb. Theory A 119 n1, (2012) 183-211.

[7] J. Desarménien. An algorithm for the Rota straightening formula, Discrete M. 30 (1980) 51-68.

[8] H. Garnir, Théorie de la représentation des groupes symétriques, Mémoires de la Soc. Royale des Sc. de Liège 10 (1950).

[9] P. Hoefsmit, Representations of Hecke algebras of finite groups with BNpairs of classical type, PhD Thesis, University of British Columbia (1974).

[10] G. James, A. Kerber. The representation theory of the symmetric group, Encyclopedia of Maths, vol 16 Addison-Wesley (1981).

[11] A.A. Jucys. On the Young operators on the symmetric groups, Lietuvos fizikos rinkinys VI:2 (1966) 163-180; Factorization of Young projection operators for the symmetric group, ibidem, X1:1 (1971) 5-10.

[12] M. Kasatani, V. Pasquier. On polynomials interpolating between the stationary state of a $O(n)$ model and a Q.H.E. ground state, Comm. Math. Phys. 276 (2007) 397-435. 
[13] D. Kazhdan and G. Lusztig. Representations of Coxeter groups and Hecke algebras, Inv. Math. (1970) 165-184.

[14] F. Knop. Symmetric and non-symmetric quantum Capelli polynomials. Comment. Math. Helvet., 72 (1997) 84-100.

[15] A. Lascoux \& M.P. Schützenberger. Symmetry and Flag manifolds, Invariant Theory, Springer L.N., 996 (1983) 118-144.

[16] A. Lascoux \& M.P. Schützenberger. Symmetrization operators on polynomial rings, Funk. Anal. 21 (1987) 77-78.

[17] A. Lascoux. Young's natural idempotents as polynomials, Annals of Combinatorics 1 (1997) 91-98.

[18] A. Lascoux. Yang-Baxter graphs, Jack and Macdonald polynomials, Tianjin 1999, Ann. Comb. 5 (2001) 397-424

[19] A. Lascoux. Symmetric Functions and Combinatorial Operators on Polynomials, CBMS/AMS Lecture Notes 99 (2003).

[20] A. Lascoux. Pfaffians and Representations of the Symmetric Group, Acta Mathematica Sinica 25 (2009) 1929-1950.

[21] D.E. Littlewood, The Theory of Group Characters and Matrix Representations of Groups, Oxford University Press, New York 1940.

[22] G.E. Murphy. On the representation theory of the symmetric groups and associated Hecke algebras, J. Algebra 152 (1992) 492-513.

[23] O. Okounkov, A.M. Vershik. A new approach to representation theory of symmetric groups, Selecta Math., 2 (1996) 581-605.

[24] G.C. Rota. Combinatorial Theory and Invariant Theory, Notes by L.Guibas, Bowdoin College (1971).

[25] D.E. Rutherford, Substitutional Analysis, Edinburgh, at the University Press, 1948.

[26] S. Sahi. Interpolation, integrality and a generalization of Macdonald's polynomials. Int. Math. Res. Not., 10 (1996) 457-471.

[27] W. Specht. Die irreduziblen darstellungen der symmetrischen gruppe, Math. Z. 39 (1935) 606-711. 
[28] R.M. Thrall. Young's semi normal representation of the symmetric group, Duke M. 8 (1941) 611-624.

[29] A. Vershik. Local algebras and a new version of Young's orthonormal form, Topics in Algebra, Banach Center Pub., 26 (1990) 467-473.

[30] H. Weyl, The classical groups, their invariants and their representations, Princeton University Press, Princeton, 1946.

[31] C.N. Yang. Some exact results for the many-body problem in one dimension with repulsive delta-function interaction, Phys. Rev. Lett. 19 (1967), $1312-1315$.

[32] A. Young. The Collected Papers of Alfred Young, University of Toronto Press (1977).

CNRS, IGM, Université de Paris-Est 77454 Marne-la-Vallée CEDEX 2

Alain.Lascoux@univ-mlv.fr

http://phalanstere.univ-mlv.fr/ al 